\title{
Theory of resonant x-ray emission spectra in compounds with localized f electrons
}

\author{
Jindřich Kolorenč \\ Institute of Physics, The Czech Academy of Sciences, Na Slovance 2, 18221 Praha, Czech Republic
}

\begin{abstract}
I discuss a theoretical description of the resonant x-ray emission spectroscopy (RXES) that is based on the Anderson impurity model. The parameters entering the model are determined from material-specific LDA+DMFT calculations. The theory is applicable across the whole $\mathrm{f}$ series, not only in the limits of nearly empty ( $\mathrm{La}, \mathrm{Ce}$ ) or nearly full ( $\mathrm{Yb}$ ) valence f shell. Its performance is illustrated on the pressure-enhanced intermediate valency of elemental praseodymium. The obtained results are compared to the usual interpretation of RXES, which assumes that the spectrum is a superposition of several signals, each corresponding to one configuration of the $4 \mathrm{f}$ shell. The present theory simplifies to such superposition only if nearly all effects of hybridization of the $4 \mathrm{f}$ shell with the surrounding states are neglected. Although the assumption of negligible hybridization sounds reasonable for lanthanides, the explicit calculations show that it substantially distorts the analysis of the RXES data.
\end{abstract}

Keywords: RXES, RIXS, dynamical mean-field theory, Anderson impurity model, intermediate valency, praseodymium

\section{Valence histogram and its measurements by core-level spectroscopy}

The $4 \mathrm{f}$ electrons in solids are typically localized and behave almost as if they were in a free atom. In some cases, the position of the $4 \mathrm{f}$ level relative to the other electronic states in a crystal is such that two configurations, say $4 \mathrm{f}^{m}$ and $4 \mathrm{f}^{m+1}$, are nearly degenerate. Then the $4 \mathrm{f}$ shell is in a mixed state referred to as the intermediate valency [1]. A related mechanism that induces an admixture of several configurations in a single 4f shell is hybridization with the other electronic states. Although this hybridization is often essentially negligible at ambient conditions, it can be enhanced by lattice compression that eventually leads to delocalization of the $4 \mathrm{f}$ states [2]. In general, the hybridization mixes more than two $4 \mathrm{f}^{m}$ configurations, each contributing with some weight $w_{m}$. The width of the histogram defined by these weights, the so-called valence histogram, then reflects the strength of the hybridization in a given compound.

The resonant x-ray emission spectroscopy (RXES, also known as the core-to-core resonant inelastic x-ray scattering, RIXS) is a direct probe of unoccupied electronic states. A measurement, in which the energy $\hbar \omega_{1}$ of the incident photons is scanned across the $\mathrm{L}$ edge, maps the $5 \mathrm{~d}$ density of states. See Fig. 1: an electron from the $2 \mathrm{p}_{3 / 2}$ core level is photoexcited to an empty $5 d_{5 / 2}$ state $\left(L_{3}\right.$ edge) and this excitation is followed by relaxation processes that fill the $2 \mathrm{p}_{3 / 2}$ core hole. In the particular setup shown in Fig. 1, a detector is tuned to monitor the photoemission at the $\mathrm{L}_{\alpha}$ line $\left(2 \mathrm{p}_{3 / 2} \rightarrow 3 \mathrm{~d}_{5 / 2}\right)$. Although the $4 \mathrm{f}$ states are not directly involved in the excitations, they do influence the measured spectra. The localized $4 \mathrm{f}$ electrons are attracted to the localized core hole, which modifies the total energy. This modification is different for

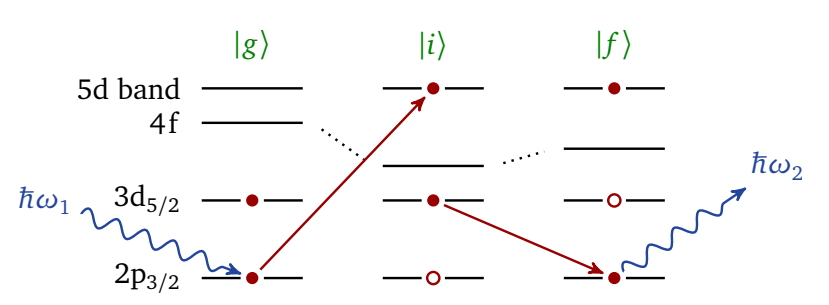

Figure 1: A cartoon of the RXES process in a lanthanide atom: an absorption of a photon $\hbar \omega_{1}$ to the ground state $|g\rangle$ is followed by an emission of a photon $\hbar \omega_{2}$ from the intermediate state $|i\rangle$. Due to the Coulomb attraction of the core hole, the $4 \mathrm{f}$ level is pulled down in energy by $U_{2 \mathrm{p} 4 \mathrm{f}}$ in the intermediate state $|i\rangle$ and by $U_{3 \mathrm{~d} 4 \mathrm{f}}$ in the final state $|f\rangle$.

different $4 \mathrm{f}^{m}$ configurations and hence the absorption edge splits when more configurations are mixed in the initial state (Fig. 2). The measured RXES spectra thus yield an information about the valence histogram [3, 4].

The pressure evolution of the valence histogram in elemental lanthanides was theoretically investigated with the aid of material-specific dynamical-mean-field theory (DMFT) [2]. Subsequently, RXES measurements at the L edge of praseodymium were performed [5], yielding a very good agreement with the theory. Using a theoretical method very similar to [2] it was argued that elemental americium approaches intermediate-valence regime when compressed [6] but L-edge RXES experiments did not detect any sign of this effect [7], possibly due to a limited resolution. Since then, more L-edge RXES measurements of actinide compounds were made [8, 9] where extraction of the valence histogram was possible. Some of the conclusions of these actinide measurements were challenged by RXES data recently collected at the M edges [10]. Indeed, the interpretation of the spectra in [8, 9] (as well as in [5]) assumed a very weak hybridization of the va- 


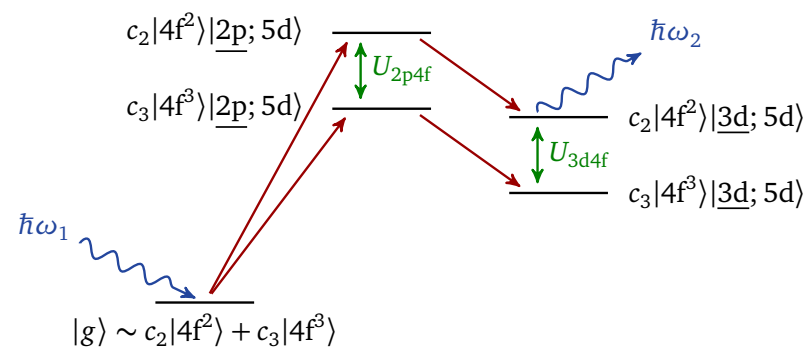

Figure 2: Total-energy diagram corresponding to Fig. 1 When the ground state of the $4 \mathrm{f}$ shell is a linear combination of two configurations (the amplitudes $c_{m}$ are given as $\sqrt{w_{m}}$ ), the intermediate and final many-electron states split due to the core-valence Coulomb interactions. Underlining indicates a hole in the particular single-electron level.

lence f shell with the surrounding states, which is not likely to be very accurate in actinides.

In this paper, I formulate a theory of RXES that does not make the assumption of weak hybridization. It generalizes the method previously used for interpreting RXES measurements on cerium and its compounds [11-13] to localized f shells with more electrons. In addition, I extract a large part of the parameters entering this theory from first-principles calculations. I apply this approach to the elemental praseodymium under pressure where both theory [2] and experiment [5] are available for comparison.

\section{Electronic structure of praseodymium under pressure}

Praseodymium crystallizes in the dhcp structure at ambient conditions. When compressed at room temperature, the crystal structure successively changes to fcc at about $5 \mathrm{GPa}$, to distorted fcc at $10 \mathrm{GPa}$, and to orthorombic at $20 \mathrm{GPa}$. The transition at $20 \mathrm{GPa}$ is accompanied by an approximately $12 \%$ volume collapse [14, 15].

All calculations presented in this paper are performed for the fcc lattice (space group Fm $\overline{3} \mathrm{~m}$ ). Since the difference between the fcc and distorted fcc phases is very small (the $c / a$ ratio in the distorted structure is only $2 \%$ or less larger than in the ideal close-packed lattice [14]), this setup covers the pressure range from 5 to $20 \mathrm{GPa}$. The lattice constants corresponding to several pressures are listed in Tab. 1 .

\subsection{Valence-band electronic structure: $L D A+D M F T$}

I start the investigation with all-electron calculations of the band structure in the local-density approximation (LDA) [16] taking into account scalar-relativistic effects as well as the spin-orbital coupling. I employ the WIEN2k package [17] with the following parameters: the radius of the muffin-tin spheres is $R_{\mathrm{MT}}=2.7 a_{\mathrm{B}}$, the basis-set cutoff $K_{\max }$ is defined with $R_{\mathrm{MT}} \times K_{\mathrm{max}}=10.5$, and the Brillouin zone is sampled with $8000 \mathrm{k}$ points ( $256 \mathrm{k}$ points in the irreducible wedge). The LDA bands of the $6 \mathrm{~s}, 4 \mathrm{f}$ and $5 \mathrm{~d}$ character are subsequently mapped onto a tight-binding model with the aid of the Wannier90 code [18, 19] (34 bands were included in the disentanglement procedure [20]).
The resulting model $\hat{H}_{\mathrm{LDA}}$ then serves as the basis for LDA+DMFT calculations. The LDA mean-field terms that correspond to the Coulomb interaction among the $4 \mathrm{f}$ electrons are replaced with an explicit two-body interaction vertex $\hat{U}$. This replacement yields a multiband Hubbard model $\hat{H}_{\mathrm{Hub}}=\hat{H}_{\mathrm{LDA}}+\sum_{n}\left(\hat{U}^{n}-U_{\mathrm{DC}}^{n}\right)$ where $n$ runs over all praseodymium atoms and $\hat{U}_{\mathrm{DC}}$, the so-called double-counting correction, approximates the mean-field terms to be removed [21]. The local Coulomb repulsion takes the form

$\hat{U}^{n}=\frac{1}{2} \sum_{\substack{m m^{\prime} m^{\prime \prime} \\ m^{\prime \prime \prime} \sigma \sigma^{\prime}}} U_{m m^{\prime} m^{\prime \prime} m^{\prime \prime \prime}} \hat{f}_{m \sigma}^{n \dagger} \hat{f}_{m^{\prime} \sigma^{\prime}}^{n \dagger} \hat{f}_{m^{\prime \prime \prime} \sigma^{\prime}}^{n} \hat{f}_{m^{\prime \prime} \sigma}^{n}$,

where $\hat{f}_{m \sigma}^{n}$ denotes the forbital with magnetic quantum number $m$ and spin direction $\sigma$ located at lattice site $n$. The matrix $U_{m m^{\prime} m^{\prime \prime} m^{\prime \prime \prime}}$ is assumed to be independent on pressure. It is parametrized by Slater integrals $F_{0}=6.0 \mathrm{eV}, F_{2}=10.2 \mathrm{eV}$, $F_{4}=6.3 \mathrm{eV}, F_{6}=4.5 \mathrm{eV}$. The average Coulomb parameter $U \equiv F_{0}$ is set the same as in [2], the other values are taken from [22]. The average Hund exchange corresponding to the given $F_{2}, F_{4}$ and $F_{6}$ is $J=0.82 \mathrm{eV}$. The double counting can be expressed as $\hat{U}_{\mathrm{DC}}^{n}=-U_{\mathrm{H}} \hat{N}_{\mathrm{f}}^{n}$ where $\hat{N}_{\mathrm{f}}^{n}=\sum_{m \sigma} \hat{f}_{m \sigma}^{n \dagger} \hat{f}_{m \sigma}^{n}$ is the number of $4 \mathrm{f}$ electrons at site $n$. The parameter $U_{\mathrm{H}}$ is set to $U_{\mathrm{H}}=7.5 \mathrm{eV}$ at all volumes. The rationale for fixing it at a single value comes from the observation that the computed LDA occupation of the $4 \mathrm{f}$ shell $n_{\mathrm{f}}$ (projection to the Wannier functions) changes only very little with compression, from 2.29 at $30 \AA^{3}$ /atom to 2.32 at $20 \AA^{3}$ /atom. The particular value $7.5 \mathrm{eV}$ was chosen to match the experimental valence-band photoemission spectra measured at ambient conditions [23].

The Hubbard model $\hat{H}_{\text {Hub }}$ is approximately solved using the dynamical-mean-field theory, that is, the many-body effects induced by the Coulomb terms $\hat{U}^{n}-U_{\mathrm{DC}}^{n}$ are taken into account only locally by means of a site-diagonal selfenergy $\hat{\Sigma}^{n}(z)$. This selfenergy is computed in an auxiliary impurity model $\hat{H}_{\text {imp }}$ that consists of one fully interacting f shell (the impurity) at site $i$ embedded in a self-consistent mean-field medium $\hat{H}_{\mathrm{MF}}=\hat{H}_{\mathrm{LDA}}+\sum_{n \neq i} \hat{\Sigma}^{n}(z)$ [24]. The impurity model can be written as $\hat{H}_{\text {imp }}=\hat{H}_{\text {imp }}^{(0)}+\hat{U}-\hat{U}_{\mathrm{DC}}$ where $\hat{H}_{\mathrm{imp}}^{(0)}$ is a noninteracting part, and the Coulomb terms $\hat{U}$ and $\hat{U}_{\mathrm{DC}}$ are the same as defined for the Hubbard model above.

Table 1: Lattice constants $a$ and lattice volumes $V$ corresponding to four investigated pressures. The data are obtained from the Murnaghan equation of state fitted to the room-temperature measurements combined from [14] and [15]. Also shown are LDA+DMFT results for the average filling of the praseodymium $4 \mathrm{f}$ shell $n_{\mathrm{f}}$ together with weights $w_{1}, w_{2}$ and $w_{3}$ of $4 \mathrm{f}^{1}, 4 \mathrm{f}^{2}$ and $4 \mathrm{f}^{3}$ configurations.

\begin{tabular}{lllll}
\hline$P(\mathrm{GPa})$ & 7.5 & 13 & 21 & \multicolumn{1}{c}{24} \\
$V /$ atom $\left(\AA^{3}\right)$ & 27.37 & 24.23 & 20.98 & 20.02 \\
$a(\AA)$ & 4.784 & 4.594 & 4.378 & 4.311 \\
$n_{\mathrm{f}}$ & 2.06 & 2.07 & 2.09 & 2.09 \\
$w_{1}$ & 0.009 & 0.015 & 0.022 & 0.023 \\
$w_{2}$ & 0.918 & 0.903 & 0.871 & 0.865 \\
$w_{3}$ & 0.072 & 0.082 & 0.105 & 0.109 \\
\hline
\end{tabular}


I neglect all non-spherical contributions (the crystal field) to $\hat{H}_{\text {imp }}^{(0)}$, which is then diagonal in the $\left|j, j_{z}\right\rangle$ basis and reads as

$$
\begin{aligned}
\hat{H}_{\mathrm{imp}}^{(0)}=\sum_{j, j_{z}} \epsilon_{j} \hat{f}_{j j_{z}}^{\dagger} \hat{f}_{j j_{z}}+\sum_{k=1}^{3} \sum_{j, j_{z}} \epsilon_{k j} \hat{b}_{k j j_{z}}^{\dagger} \hat{b}_{k j j_{z}} \\
+\sum_{k=1}^{3} \sum_{j, j_{z}} V_{k j}\left(\hat{f}_{j j_{z}}^{\dagger} \hat{b}_{k j j_{z}}+\hat{b}_{k j j_{z}}^{\dagger} \hat{f}_{j j_{z}}\right)
\end{aligned}
$$

The orbitals $\hat{f}_{j j_{z}}$ belong to the impurity f shell, the orbitals $\hat{b}_{k j j_{z}}$ constitute the non-interacting medium (the so-called bath). Optimally, the model would contain an infinite number of bath orbitals ( $k$ would run to $\infty$ ) to fully represent $\hat{H}_{\mathrm{MF}}$ in an infinite lattice. The restriction to a finite bath with $1 \leq k \leq 3$ is a limitation of the exact-diagonalization method that I employ to solve the model. The comparison to experiment will provide a justification for the finite bath in the present application. More thorough discussion of accuracy of the exact diagonalization in the context of LDA+DMFT can be found elsewhere [25].

The parameters $\epsilon_{j}, \epsilon_{k j}$ and $V_{k j}$ that enter Eq. (2) are determined by matching the f-shell matrix elements of the oneparticle Green's function computed from $\hat{H}_{\mathrm{imp}}^{(0)}$,

$G_{\mathrm{f}, j}^{(0)}\left(\mathrm{i} \omega_{n}\right)=\left(z-\epsilon_{j}-\sum_{k=1}^{3} \frac{V_{k j}^{2}}{\mathrm{i} \omega_{n}-\epsilon_{k j}}\right)^{-1}$,

to the f-shell matrix elements of the so-called bath Green's function $\mathscr{G}_{j}$ that corresponds to the mean-field medium $\hat{H}_{\mathrm{MF}}$ [24]. This matching is implemented as a minimization of a cost function in the form

$\chi_{j}=\sum_{n=n_{\min }}^{n_{\max }}\left|\frac{1}{G_{\mathrm{f}, j}^{(0)}\left(\mathrm{i} \omega_{n}\right)}-\frac{1}{\mathscr{G}_{j}\left(\mathrm{i} \omega_{n}\right)}\right|^{2}$

where $\omega_{n}$ are Matsubara frequencies [26]. I introduce a lower cutoff $n_{\min }$ such that the smallest Matsubara frequency included in the fit is $\omega_{n} \approx 3 \mathrm{eV}$. That way, the high-energy asymptotics of $G_{\mathrm{f}, j}^{(0)}$ and $\mathscr{G}_{j}$ are as close as possible to each other, which is advantageous for calculation of thermodynamic quantities such as the total energy [24]. Although my goal is spectra, the spectra in question do not involve the $4 \mathrm{f}$ states directly, and it is the total energy of the $4 \mathrm{f}$ shell that plays the important role (Fig. 2).

During the DMFT calculations, the selfenergy $\hat{\Sigma}(z)$ of the impurity model $\hat{H}_{\text {imp }}$ is computed using an in-house exact-diagonalization code [27] that combines the implicitly restarted Lanczos method for finding the many-body spectrum [28] with the band Lanczos method for evaluation of the oneparticle Green's function [29]. The computational demands are reduced with the aid of a truncated Hilbert space [11, 27]. If the basis states are denoted as $\left|f^{r} b^{n} \underline{b}^{m}\right\rangle$, where $r$ indicates the number of electrons in the f states, $\bar{n}$ the number electrons in the bath states above the Fermi level, and $m$ the number of

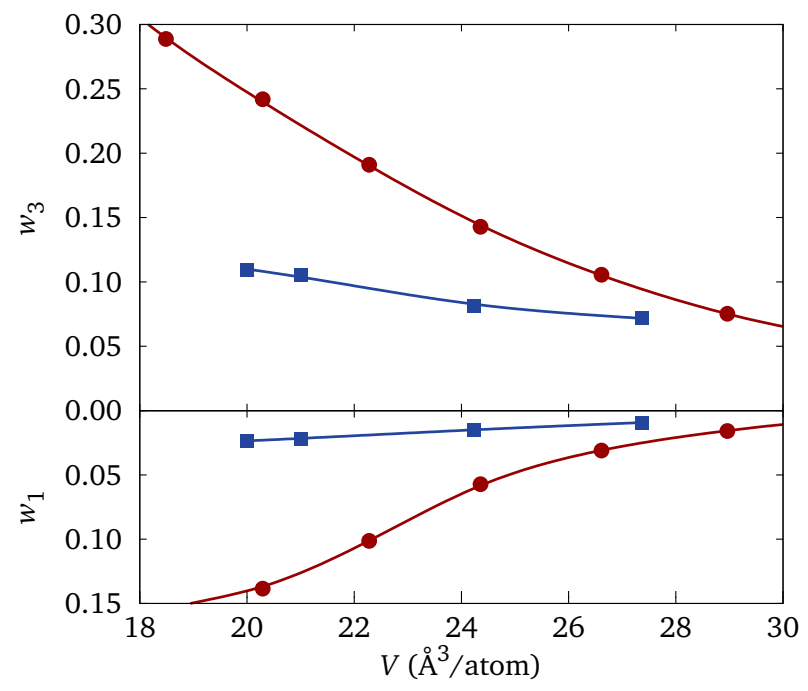

Figure 3: The configuration weights $w_{1}$ and $w_{3}$ defined in Eq. (6) as functions of volume. The present results (blue squares) are compared to earlier calculations (red points) [2].

holes in the bath states below the Fermi level, the truncated $N$-electron Hilbert space is defined as

$\mathscr{H}_{N}^{(M)}=\left\{\left|f^{N-N_{b}^{<-n+m}} b^{n} \underline{b}^{m}\right\rangle, 0 \leq m+n \leq M\right\}$.

Here $N_{b}^{<}$is the number of bath orbitals below the Fermi level and the parameter $M$ controls the size of the Hilbert space. All calculations reported in this paper correspond to $M=2$.

The results of the LDA+DMFT calculations are summarized in Fig. 3 and in Tab. 1. The table lists the pressure evolution of the valence histogram

$w_{m}=\left\langle\psi_{0}\left|\delta\left(\hat{N}_{\mathrm{f}}-m\right)\right| \psi_{0}\right\rangle, \quad m=0,1,2, \ldots$

that comprises weights $w_{m}$ of the individual $4 \mathrm{f}^{m}$ configurations that contribute to the ground state $\left|\psi_{0}\right\rangle$ of the impurity model $\hat{H}_{\text {imp }}$. The average filling of the $4 \mathrm{f}$ shell, also included in Tab. 1, can then be written as $n_{\mathrm{f}}=\left\langle\psi_{0}\left|\hat{N}_{\mathrm{f}}\right| \psi_{0}\right\rangle=\sum_{m} m w_{m}$. Figure 3 shows the pressure dependence of $w_{1}$ and $w_{3}$ that measure the departure from the $4 \mathrm{f}^{2}$ atomic limit. The present results are compared to the earlier LDA+DMFT calculations [2]. Apparently, there is a large discrepancy, which I will return to in Sec. 3 .

\subsection{Resonant $x$-ray emission spectra}

The resonant $\mathrm{x}$-ray emission spectroscopy can be simulated in an impurity model

$$
\begin{aligned}
\hat{H}=\hat{H}_{\text {imp }}+\epsilon_{2 \mathrm{p}} \hat{p}^{\dagger} \hat{p}+\epsilon_{3 \mathrm{~d}} \hat{d}^{\dagger} \hat{d}+\sum_{k} \epsilon_{k} \hat{d}_{k}^{\dagger} \hat{d}_{k} \\
+\left[U_{2 \mathrm{p} 4 \mathrm{f}}\left(\hat{p}^{\dagger} \hat{p}-1\right)+U_{3 \mathrm{~d} 4 \mathrm{f}}\left(\hat{d}^{\dagger} \hat{d}-1\right)\right] \hat{N}_{\mathrm{f}}
\end{aligned}
$$

that represents an extension of the impurity model $\hat{H}_{\text {imp }}$ that appeared in Sec. 2.1 as a part of the LDA+DMFT theory. The model $\hat{H}_{\text {imp }}$ is supplemented with the following orbitals located at the impurity site: the $2 \mathrm{p}_{3 / 2}$ core state $\hat{p}$, the $3 \mathrm{~d}_{5 / 2}$ 
core state $\hat{d}$, and the $5 d_{5 / 2}$ band $\hat{d}_{k}$. The core electrons and the $4 \mathrm{f}$ electrons are localized, and they repel each other by the Coulomb interaction parametrized by $U_{2 \mathrm{p} 4 \mathrm{f}}$ for the $2 \mathrm{p}_{3 / 2}$ state and by $U_{3 \mathrm{~d} 4 \mathrm{f}}$ for the $3 \mathrm{~d}_{5 / 2}$ state. The degeneracy of the core levels is neglected and hence there is only one core-valence Slater integral. The interaction between the $4 \mathrm{f}$ electrons and $5 \mathrm{~d}$ electrons can be neglected thanks to the delocalized character of the $5 \mathrm{~d}$ states.

The cross section of the RXES process is approximated by the Kramers-Heisenberg formula [3, 30]

$$
\begin{aligned}
\sigma\left(\omega_{1}, \omega_{2}\right)=\sum_{f}\left|\sum_{i} \frac{\left\langle f\left|\hat{T}_{2}\right| i\right\rangle\left\langle i\left|\hat{T}_{1}\right| g\right\rangle}{E_{i}-E_{g}-\omega_{1}-\mathrm{i} \Gamma_{2 \mathrm{p}}}\right|^{2} \\
\times \frac{\Gamma_{3 \mathrm{~d}} / \pi}{\left(\omega_{1}-\omega_{2}-E_{f}+E_{g}\right)^{2}+\Gamma_{3 \mathrm{~d}}^{2}}
\end{aligned}
$$

where $|g\rangle$ is the initial ground state with completely filled core states and empty $5 \mathrm{~d}$ states, $|i\rangle$ is an intermediate state with a $2 \mathrm{p}_{3 / 2}$ core hole and an excited electron in the $5 \mathrm{~d}$ band, and $|f\rangle$ is a final state with a $3 \mathrm{~d}_{5 / 2}$ core hole and one $5 \mathrm{~d}$ electron. The total energies of these states are denoted as $E_{g}, E_{i}$ and $E_{f}$; $\Gamma_{2 p}$ and $\Gamma_{3 \mathrm{~d}}$ are half widths at half maximum of the $2 \mathrm{p}_{3 / 2}$ and $3 \mathrm{~d}_{5 / 2}$ hole states, and $\omega_{1}$ and $\omega_{2}$ stand for energies of the incident and emitted photons. Finally, $\left\langle f\left|\hat{T}_{2}\right| i\right\rangle$ and $\left\langle i\left|\hat{T}_{1}\right| g\right\rangle$ are the dipole matrix elements. If I consider only the angular part of the dipole operator, I have $\hat{T}_{1}=\sum_{k} \hat{d}_{k}^{\dagger} \hat{p}$ and $\hat{T}_{2}=\hat{p}^{\dagger} \hat{d}$.

The initial state can be written as $|g\rangle=\hat{p}^{\dagger} \hat{d}^{\dagger}\left|\psi_{0}\right\rangle$ where $\left|\psi_{0}\right\rangle$ is the correlated ground state of the impurity model $\hat{H}_{\text {imp }}$, $\hat{H}_{\text {imp }}\left|\psi_{0}\right\rangle=E_{0}\left|\psi_{0}\right\rangle$. Apparently, $|g\rangle$ is an eigenstate of the full hamiltonian $\hat{H}$, that is, $\hat{H}|g\rangle=E_{g}|g\rangle$ with $E_{g}=E_{0}+\epsilon_{2 \mathrm{p}}+\epsilon_{3 \mathrm{~d}}$. The Kramers-Heisenberg formula as written in Eq. (8) is useful when it is possible to explicitly calculate all intermediate and final states as eigenstates of the hamiltonian $\hat{H}$. This is not the case in the present application since the Hilbert space is too large. For the purposes of the Krylov-subspace method, which I intend to use, I rewrite Eq. (8) into an operator form.

First, I can replace the sums $\sum_{i}$ and $\sum_{f}$ with sums over all eigenstates of $\hat{H}$ since the transition operators $\hat{T}_{1}$ and $\hat{T}_{2}$ will select the right states anyway,

$$
\begin{aligned}
& \sigma\left(\omega_{1}, \omega_{2}\right)=\sum_{k}\left\langle\psi_{0}\right| \hat{d} \hat{d}_{k} \frac{1}{\hat{H}-E_{g}-\omega_{1}+\mathrm{i} \Gamma_{2 \mathrm{p}}} \hat{d}^{\dagger} \hat{p} \\
& \times \frac{\Gamma_{3 \mathrm{~d}} / \pi}{\left(\omega_{1}-\omega_{2}-\hat{H}+E_{g}\right)^{2}+\Gamma_{3 \mathrm{~d}}^{2}} \\
& \times \hat{p}^{\dagger} \hat{d} \frac{1}{\hat{H}-E_{g}-\omega_{1}-\mathrm{i} \Gamma_{2 \mathrm{p}}} \hat{d}_{k}^{\dagger} \hat{d}^{\dagger}\left|\psi_{0}\right\rangle .
\end{aligned}
$$

The fermionic operators associated with the uncorrelated states can be commuted toward $\left\langle\psi_{0}\right|$ where they all annihilate. Along the way, the full impurity hamiltonian $\hat{H}$ is replaced by two smaller hamiltonians,

$$
\begin{aligned}
\sigma\left(\omega_{1}, \omega_{2}\right)= & \sum_{k}\left\langle\psi_{0}\right| \frac{1}{\hat{H}_{\mathrm{imp}}^{\prime}-E_{0}-\epsilon_{2 \mathrm{p}}+\epsilon_{k}-\omega_{1}+\mathrm{i} \Gamma_{2 \mathrm{p}}} \\
& \times \frac{\Gamma_{3 \mathrm{~d}} / \pi}{\left(\omega_{1}-\omega_{2}-\hat{H}_{\mathrm{imp}}^{\prime \prime}+E_{0}+\epsilon_{3 \mathrm{~d}}-\epsilon_{k}\right)^{2}+\Gamma_{3 \mathrm{~d}}^{2}} \\
& \times \frac{1}{\hat{H}_{\mathrm{imp}}^{\prime}-E_{0}-\epsilon_{2 \mathrm{p}}+\epsilon_{k}-\omega_{1}-\mathrm{i} \Gamma_{2 \mathrm{p}}}\left|\psi_{0}\right\rangle,
\end{aligned}
$$

where $\hat{H}_{\text {imp }}^{\prime}=\hat{H}_{\text {imp }}-U_{2 \mathrm{p} 4 \mathrm{f}} \hat{N}_{\mathrm{f}}$ and $\hat{H}_{\text {imp }}^{\prime \prime}=\hat{H}_{\text {imp }}-U_{3 \mathrm{~d} 4 \mathrm{f}} \hat{N}_{\mathrm{f}}$. These hamiltonians correspond to the intermediate and final states where the $4 \mathrm{f}$ level is pulled down by $U_{2 \mathrm{p} 4 \mathrm{f}}$ and $U_{3 \mathrm{~d} 4 \mathrm{f}}$ due to interaction with the core hole (Fig. 1).

The cross section $\sigma\left(\omega_{1}, \omega_{2}\right)$ takes the form of a diagonal matrix element of a product of three functions of two noncommuting operators, which is a rather complex object. If, however, the two hamiltonians $\hat{H}_{\mathrm{imp}}^{\prime}$ and $\hat{H}_{\mathrm{imp}}^{\prime \prime}$ were the same, that is, if $U_{2 \mathrm{p} 4 \mathrm{f}}=U_{3 \mathrm{~d} 4 \mathrm{f}}=U_{\mathrm{cv}}$, the formula would reduce to an expectation value of a function of a single operator, $\left\langle\psi_{0}\left|F\left(\hat{H}_{\mathrm{imp}}^{\prime}\right)\right| \psi_{0}\right\rangle$. Such matrix element is considerably simpler and can be evaluated with the aid of the Krylov-subspace approximation, see Appendix A The assumption of equal core-valence Coulomb interactions $U_{\mathrm{cv}}$ in the intermediate and final states is well justified in the case of the deep 2p and $3 \mathrm{~d}$ core states. In cerium, $U_{2 \mathrm{p} 4 \mathrm{f}}$ and $U_{3 \mathrm{~d} 4 \mathrm{f}}$ differ only by about $0.5 \mathrm{eV}$ [13].

Finally, I replace the momentum summation in Eq. (10) with an integral over $5 \mathrm{~d}$ single-particle energies, which yields a convolution of a many-body expectation value with the density of $5 d_{5 / 2}$ states that is taken from the LDA+DMFT calculations,

$$
\begin{aligned}
\sigma=\int & \mathrm{d} \epsilon g_{5 \mathrm{~d}_{5 / 2}}(\epsilon)\left\langle\psi_{0}\right| \frac{1}{\left(\hat{H}_{\mathrm{imp}}^{\prime}-E_{0}-\epsilon_{2 \mathrm{p}}+\epsilon-\omega_{1}\right)^{2}+\Gamma_{2 \mathrm{p}}^{2}} \\
& \times \frac{\Gamma_{3 \mathrm{~d}} / \pi}{\left(\omega_{1}-\omega_{2}-\hat{H}_{\mathrm{imp}}^{\prime}+E_{0}+\epsilon_{3 \mathrm{~d}}-\epsilon\right)^{2}+\Gamma_{3 \mathrm{~d}}^{2}}\left|\psi_{0}\right\rangle .
\end{aligned}
$$

The spectra given by Eq. (11) are plotted in Figs. 4 and 5 , The parameters not fixed by the first-principles calculations (Sec. 2.1) are the core-valence Coulomb parameter, which is assumed to be $25 \%$ larger than the valence-valence Coulomb parameter, $U_{\mathrm{cv}}=1.25 \mathrm{U}=7.5 \mathrm{eV}$, and the line widths $\Gamma_{2 \mathrm{p}}=5.0 \mathrm{eV}$ and $\Gamma_{3 \mathrm{~d}}=1.5 \mathrm{eV}$ that combine the natural line width and the instrument resolution $\left(\Gamma_{2 p}\right.$ is possibly overestimated [31]).

Figure 4 shows $\sigma\left(\omega_{1}, \omega_{2}\right)$ at $7.5 \mathrm{GPa}$ for incident-photon energy $\omega_{1}$ approaching the absorption edge. One can see how the signal corresponding to the minority $4 \mathrm{f}^{3}$ configuration is resonantly enhanced before the main edge corresponding to $4 \mathrm{f}^{2}$ is reached. Compared to experiment [5], the absolute position of the $\mathrm{L}_{3}$ edge is estimated poorly, but the shapes of the spectra for incident energies at comparable distances from the edge are reproduced rather accurately. Figure 5 shows RXES spectra at different pressures, all calculated for $\omega_{1}=5827 \mathrm{eV}$, that is, about $16 \mathrm{eV}$ below the maximum of the absorption line. Again, the spectra look like those experimentally observed. 

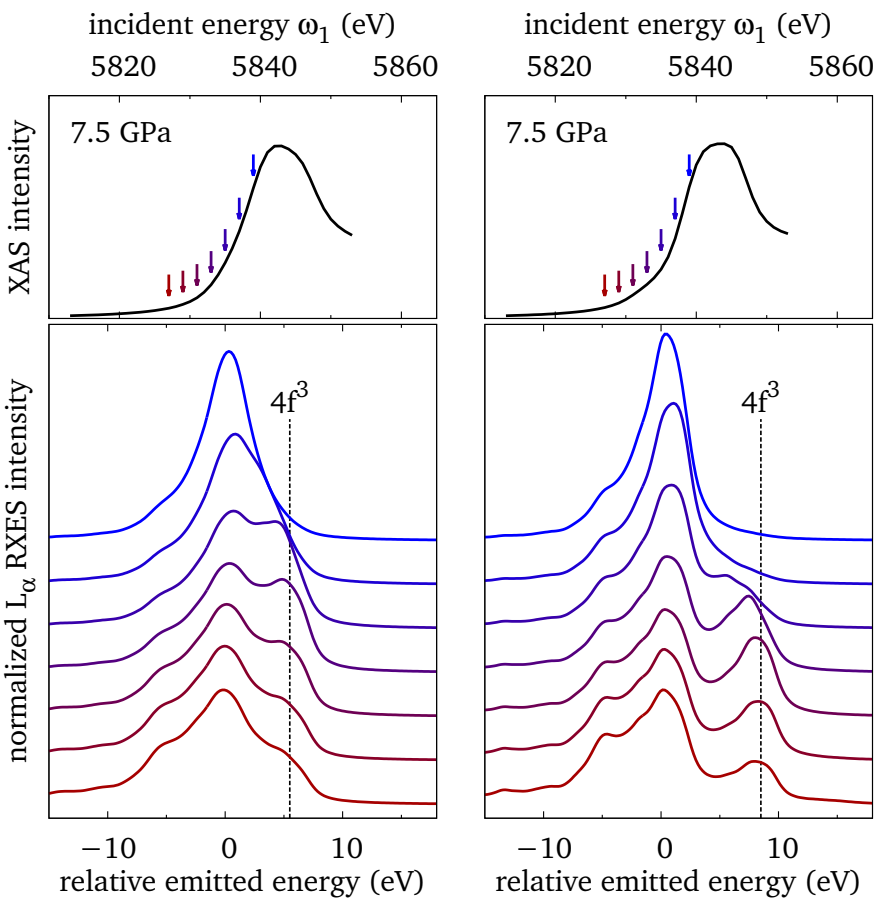

Figure 4: Theoretical RXES spectra corresponding to the incident energies $\omega_{1}$ approaching the $\mathrm{L}_{3}$ absorption edge (top panel). The relative emitted energy on the horizontal axis means $\omega_{2}-\omega_{1}$ shifted so that the main peak is centered at zero. Left: The theory given by Eq. (11) closely matches the experimental data shown in Fig. 1 of [5]. Right: The simplified theory represented by Eqs. (12) is significantly worse.

Note, however, that the experimental data shown in Fig. 2a of [5] are taken at the incident energy closer to the maximum of the absorption line.

\section{Discussion}

The theoretical approach outlined in Sec. 2 accurately reproduces the RXES spectra experimentally measured for elemental praseodymium [5]. It does so with a $4 \mathrm{f}$ valence histogram substantially different from the histogram previously calculated in [2] as well as from the histogram deduced from the experimental data in [5]. The latter two are compatible to each other, the present theory is at odds with both, see Tab. 2 for details. My calculations suggest that a considerably smaller departure from the $4 \mathrm{f}^{2}$ atomic limit than previously thought is needed to explain the measured RXES.

The theory used in [2] is very similar to the method discussed here in Sec. 2.1. It is also LDA+DMFT, only: (i) the impurity model is solved by the Hirsch-Fye quantum Monte Carlo (QMC) instead of the exact diagonalization, (ii) the Hund exchange is neglected, that is, $F_{2}=F_{4}=F_{6}=0$, and (iii) the Coulomb parameter $U$ is volume dependent [32]. The reduction of $U$ due to more efficient screening in a compressed lattice certainly causes some increase of $w_{1}$ and $w_{3}$, but hardly as much as would be needed to explain the large discrepancy shown in Fig. 3 and Tab. 2 since $U$ drops only by $0.5 \mathrm{eV}$ from $30 \AA^{3}$ /atom to $20 \AA^{3}$ /atom [32]. A more substantial issue is the calculation of the weights $w_{m}$. Unlike certain variants of

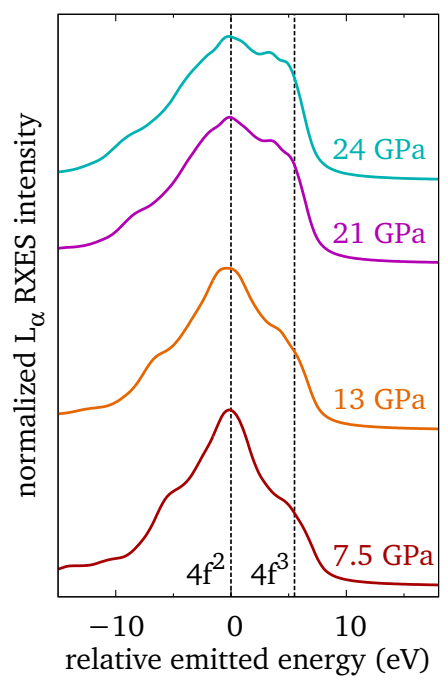

Figure 5: RXES spectrum as a function of pressure. The shoulder associated with the $4 \mathrm{f}^{3}$ component grows in a compressed lattice. The theoretical curves accurately reproduce the measurements shown in Fig. 2a of [5].

the continuous-time QMC [33], the Hirsch-Fye QMC does not provide a direct access to these weights, which were estimated using expressions borrowed from the atomic limit, thus neglecting hybridization [2].

An assumption of vanishingly small hybridization was employed also when the RXES measurements were analyzed in [5]: the spectra were fitted by a linear combination of two signals, and their intensities were directly interpreted as the weights $w_{2}$ and $w_{3}$. The cross section derived in Sec. 2.2 displays such a simple behavior only if: (i) the hybridization is neglected in $\hat{H}_{\mathrm{imp}}^{\prime}$, which corresponds to the condition $U_{\mathrm{cv}} \gg V_{k j}$, and (ii) the incident energy is far below the absorption edge, that is, $\epsilon_{2 p}+\omega_{1}$ is much larger than $U_{\mathrm{cv}}, \Gamma_{2 \mathrm{p}}$ and the $5 \mathrm{~d}$ bandwidth. Under these conditions, Eq. (11) reduces to

$\sigma\left(\omega_{1}, \omega_{2}\right)=\sum_{m} w_{m} f\left(\omega_{1}-\omega_{2}+m U_{\mathrm{cv}}\right)$

where

$f(x)=\int \mathrm{d} \epsilon \frac{g_{5 \mathrm{~d}_{5 / 2}}(\epsilon)}{\left(\epsilon_{2 \mathrm{p}}+\omega_{1}\right)^{2}} \frac{1}{\left(x-\epsilon+\epsilon_{3 \mathrm{~d}}\right)^{2}+\Gamma_{3 \mathrm{~d}}^{2}}$.

To gauge the consequences of neglecting the hybridization in the intermediate and final states of RXES, the spectra calculated with the simplified formula and with Eq. (11) are

Table 2: The weight of the $4 \mathrm{f}^{3}$ configuration in the ground state increases with pressure. The present theory (DMFT/ED) suggests a noticeably slower growth than both the earlier theory (DMFT/QMC) [2] and the earlier interpretation of the measured RXES data [5].

\begin{tabular}{cccc}
\hline \multirow{2}{*}{$P(\mathrm{GPa})$} & \multicolumn{3}{c}{$w_{3} / w_{2}$} \\
\cline { 2 - 4 } & DMFT/ED & DMFT/QMC [2] & exp. [5] \\
\hline 24 & 0.126 & 0.42 & $0.46(5)$ \\
21 & 0.121 & 0.34 & $0.33(5)$ \\
13 & 0.090 & 0.19 & $0.18(5)$ \\
7.5 & 0.079 & 0.11 & $0.13(5)$ \\
\hline
\end{tabular}


compared in Fig. 4 for otherwise identical parameters. The differences are sizable.

Both the experiment [5] and the earlier theory [2] assume somewhere along the way that the hybridization of the $4 \mathrm{f}$ shell with the surrounding electronic states is negligibly small, although it is exactly this hybridization that is the dominant cause of the investigated effect: the nonzero weights $w_{1}$ and $w_{3}$. The present theory does not rely on this assumption.

\section{Summary}

I have outlined a theoretical model for the resonant x-ray emission spectra measured at the L edge in f-electron compounds. It builds on the ideas developed for Ce compounds in the past [11, 12] and extends them to take into account the full multiplet structure of the valence $f$ states. The theory avoids the assumption of very weak hybridization of the f shell with the surrounding states, which is typically made when interpreting the experimental spectra [5, 8, 9]. Taking compressed praseodymium as an example, I have illustrated that this assumption can skew the determination of the valence histogram already in lanthanides, with the experiments on actinides being affected even more.

\section{Acknowledgments}

This work was supported by the Czech Science Foundation [grant number 15-05872J]. Access to computing facilities owned by parties and projects contributing to the National Grid Infrastructure MetaCentrum, provided under the program Cesnet LM2015042, is appreciated.

\section{Appendix A. Krylov-subspace approximation}

A diagonal matrix element of a function of an operator $\hat{A}$ can be approximated as [29]

$$
\langle\psi|f(\hat{A})| \psi\rangle=\langle\psi|f(\hat{B})| \psi\rangle+\mathrm{O}\left(\hat{A}^{n+1}\right)
$$

where $\hat{B}$ is an operator living in a Krylov subspace

$\mathscr{K}_{n}=\left\{|\psi\rangle, \hat{A}|\psi\rangle, \hat{A}^{2}|\psi\rangle, \ldots, \hat{A}^{n}|\psi\rangle\right\}$

The operator $\hat{B}$ is defined as $B_{i j}=\left\langle\phi_{i}|\hat{A}| \phi_{j}\right\rangle$, where $\left\{\left|\phi_{i}\right\rangle\right\}$ is an orthonormal basis of $\mathscr{K}_{n}$ such that $\left|\phi_{1}\right\rangle=|\psi\rangle$. The method is useful if the approximation is sufficiently accurate already for $n$ so small that all eigenvalues $b_{i}$ of $\hat{B}$ and their corresponding eigenvectors $\left|b_{i}\right\rangle$ can be explicitly found, and one can use the spectral representation of Eq. (A.1),

$$
\langle\psi|f(\hat{A})| \psi\rangle=\sum_{i=1}^{n}\left|\left\langle\psi \mid b_{i}\right\rangle\right|^{2} f\left(b_{i}\right)+\mathrm{O}\left(\hat{A}^{n+1}\right) .
$$

[1] J. M. Lawrence, P. S. Riseborough, and R. D. Parks, Rep. Prog. Phys. 44, 1-84 (1981)

[2] A. K. McMahan, R. T. Scalettar, and M. Jarrell, Phys. Rev. B 80, 235105 (2009) arXiv:0909.0539 [cond-mat.str-el]

[3] A. Kotani and S. Shin, Rev. Mod. Phys. 73, 203-246 (2001)

[4] J.-P. Rueff and A. Shukla, Rev. Mod. Phys. 82, 847-896 (2010) arXiv:0812.0538 [cond-mat.str-el]

[5] J. A. Bradley, K. T. Moore, M. J. Lipp, B. A. Mattern, J. I. Pacold, G. T. Seidler, P. Chow, E. Rod, Y. Xiao, and W. J. Evans, Phys. Rev. B 85, $100102(2012)$

[6] S. Y. Savrasov, K. Haule, and G. Kotliar, Phys. Rev. Lett. 96, 036404 (2006) arXiv:cond-mat/0507552 [cond-mat.str-el]

[7] S. Heathman, J.-P. Rueff, L. Simonelli, M. A. Denecke, J.-C. Griveau, R. Caciuffo, and G. H. Lander, Phys. Rev. B 82, 201103 (2010)

[8] C. Booth, S. Medling, Y. Jiang, E. Bauer, P. Tobash, J. Mitchell, D. Veirs, M. Wall, P. Allen, J. Kas, D. Sokaras, D. Nordlund, and T.-C. Weng, J. Electron. Spectrosc. Relat. Phenom. 194, 57-65 (2014)

[9] C. H. Booth, S. A. Medling, J. G. Tobin, R. E. Baumbach, E. D. Bauer, D. Sokaras, D. Nordlund, and T.-C. Weng, Phys. Rev. B 94, 045121 (2016) arXiv:1607.03953 [cond-mat.str-el]

[10] K. O. Kvashnina, H. C. Walker, N. Magnani, G. H. Lander, and R. Caciuffo, Phys. Rev. B 95, 245103 (2017) arXiv:1706.02920 [cond-mat.str-el]

[11] O. Gunnarsson and K. Schönhammer, Phys. Rev. B 28, 4315-4341 (1983)

[12] M. Nakazawa, S. Tanaka, T. Uozumi, and A. Kotani, J. Phys. Soc. Jpn. 65, 2303-2310 (1996)

[13] J.-P. Rueff, J.-P. Itié, M. Taguchi, C. F. Hague, J.-M. Mariot, R. Delaunay, J.-P. Kappler, and N. Jaouen, Phys. Rev. Lett. 96, 237403 (2006) arXiv:cond-mat/0602169 [cond-mat.str-el]

[14] B. J. Baer, H. Cynn, V. Iota, C.-S. Yoo, and G. Shen, Phys. Rev. B 67, 134115 (2003)

[15] G. N. Chesnut and Y. K. Vohra, Phys. Rev. B 62, 2965-2968 (2000)

[16] J. P. Perdew and Y. Wang, Phys. Rev. B 45, 13244-13249 (1992)

[17] P. Blaha, K. Schwarz, G. K. H. Madsen, D. Kvasnicka, and J. Luitz, WIEN2k, An Augmented Plane Wave + Local Orbitals Program for Calculating Crystal Properties (Techn. Universität Wien, Austria, 2001).

[18] A. A. Mostofi, J. R. Yates, Y.-S. Lee, I. Souza, D. Vanderbilt, and N. Marzari, Comput. Phys. Commun. 178, 685-699 (2008) arXiv:0708.0650 [cond-mat.mtrl-sci]

[19] J. Kuneš, R. Arita, P. Wissgott, A. Toschi, H. Ikeda, and K. Held, Comput. Phys. Commun. 181, 1888-1895 (2010) arXiv:1004.3934 [condmat.mtrl-sci]

[20] I. Souza, N. Marzari, and D. Vanderbilt, Phys. Rev. B 65, 035109 (2001) arXiv:cond-mat/0108084 [cond-mat.mtrl-sci]

[21] A. I. Lichtenstein and M. I. Katsnelson, Phys. Rev. B 57, 6884-6895 (1998) arXiv:cond-mat/9707127 [cond-mat.str-el]

[22] S. Lebègue, A. Svane, M. I. Katsnelson, A. I. Lichtenstein, and O. Eriksson, Phys. Rev. B 74, 045114 (2006)

[23] J. K. Lang, Y. Baer, and P. A. Cox, J. Phys. F: Met. Phys. 11, 121-138 (1981)

[24] A. Georges, G. Kotliar, W. Krauth, and M. J. Rozenberg, Rev. Mod. Phys. 68, 13-125 (1996) arXiv:cond-mat/9510091

[25] A. Liebsch and H. Ishida, J. Phys.: Condens. Matter 24, 053201 (2012) arXiv:1109.0158 [cond-mat.str-el]

[26] M. Caffarel and W. Krauth, Phys. Rev. Lett. 72, 1545-1548 (1994)

[27] J. Kolorenč, A. B. Shick, and A. I. Lichtenstein, Phys. Rev. B 92, 085125 (2015) arXiv:1504.07979 [cond-mat.str-el]

[28] R. B. Lehoucq, D. C. Sorensen, and C. Yang, ARPACK Users' Guide, Solution of Large-Scale Eigenvalue Problems with Implicitly Restarted Arnoldi Methods (SIAM, Philadelphia, PA, 1998).

[29] H.-D. Meyer and S. Pal, J. Chem. Phys. 91, 6195-6204 (1989)

[30] J. W. Sakurai, Advanced Quantum Mechanics (Addison-Wesley, 1967).

[31] M. O. Krause and J. H. Oliver, J. Phys. Chem. Ref. Data 8, 329-338 (1979)

[32] A. McMahan, C. Huscroft, R. Scalettar, and E. Pollock, J. Comput.Aided Mater. Des. 5, 131-162 (1998) arXiv:cond-mat/9805064 [condmat.str-el]

[33] E. Gull, A. J. Millis, A. I. Lichtenstein, A. N. Rubtsov, M. Troyer, and P. Werner, Rev. Mod. Phys. 83, 349-404 (2011) arXiv:1012.4474 [condmat.str-el] 